\title{
Detection of Vehicle Mounted Auditory Reverse Alarm using Hidden Markov Model
}

\author{
Mikael Swartling, Mikael Nilsson and Nedelko Grbić \\ Department of Signal Processing \\ Blekinge Institute of Technology \\ SE-372 25 Ronneby, Sweden \\ E-mail: msw@bth.se
}

\begin{abstract}
This paper presents a method for automatically detecting vehicle mounted auditory reverse alarms, or other similar warning signals, based on hidden Markov model and pattern matching techniques. The method is designed for embedded realtime platforms. The purpose of the method is to embed it with active hearing protection devices, aiding the user in detecting warning signals in low SNR environments. Real recordings are used to evaluate the performance, and the results are presented.
\end{abstract}

Keywords - Alarm detection, Hidden Markov models, Pattern Matching.

\section{INTRODUCTION}

An important safety issue at an industrial workplace is the interaction between workers and moving vehicles. Due to limited visibility at the drivers seat in many vehicles, the risk of an accident increases when driving in reverse. Different kinds of warning systems exists to prevent accidents and an auditory reverse alarm is one such system. The auditory reverse alarm consists of a buzzer emitting a single frequency pulsating tone. The auditory reverse alarm is automatically turned on when, for example, the vehicle is in reverse gear.

At a workplace with high noise levels, hearing protection devices are often required by safety regulations. However, hearing protection devices degrades the workers detection and localization ability [1], making it difficult to both detect the reverse alarm and localize the source of the potential danger. In addition, hearing impaired workers may have even more difficulty in both detecting the alarm and localize it.

This paper presents a method to automatically detect the presence of a general single frequency tone with a pulsating pattern. It it based on hidden Markov model (HMM) and pattern matching techniques, designed for realtime platforms. The purpose of the method to embed it with active hearing protection devices to aid the users in both detecting and locating auditory alarms similar to the reverse alarms. It is computationally simple and suitable for realtime platforms such as the active hearing protection devices that exists today.

\section{SIGNAL MODEL AND FEATURES}

In the fullband signal $x(n)$ sampled at the sensor, the signal pattern to detect and match is located in a narrow frequency band. The center frequency of the pass- band is the frequency of the tone, $f_{t}$, emitted by the reverse alarm. By performing a passband sampling around $f_{t}$, a baseband representation $x_{f_{t}}(n)$ of the signal of interest is estimated.

The passband sampling is similar to a uniform DFT analysis filterbank [2], where a subband oversampling can be used to reduce the aliasing distortion [3]. The difference from a uniform DFT analysis filterbank is that only a single frequency bin is of interest, and that the center frequency can be chosen arbitrarily. A filter $h_{f_{t}}(n)$ is created as

$$
h_{f_{t}}(n)=h(n) \cdot W_{f_{t}}(n)
$$

where the modulation factor $W$ is

$$
W_{f_{t}}(n)=e^{j \cdot 2 \pi n \frac{f_{t}}{f_{s}}}
$$

and the prototype filter $h(n)$ is a lowpass FIR filter of length $K \cdot L$ with a cutoff frequency of $\frac{f_{s}}{K}$.

The fullband signal is filtered by the modulated prototype filter to get the baseband signal $x_{f_{t}}(n)$ of $x(n)$ at the frequency $f_{t}$. Because the frequency content of $x_{f_{t}}(n)$ is limited by the prototype filter to a bandwidth of $\frac{f_{s}}{K}$, the baseband signal can be decimated by a factor $K$. According to the noble identity, decimation can be performed before filtering, allowing an efficient polyphase implementation.

Assuming the bandwidth of the modulated prototype filter is small, the reverse alarm is assumed to be dominant within the passband. The baseband signal is a complex valued signal, and the magnitude is proportional to the magnitude emitted by the reverse alarm.

The magnitude of the baseband signal is the base of the feature extraction for the HMM and pattern matching. However, the actual magnitude varies depending on physical properties of the reverse alarm, sensor settings and distance of acoustic propagation. 
In order to make the features amplitude independent, an amplitude normalization step is performed. Assuming the amplitude of the pulses received at the sensor is $A$ with a duty cycle factor of $d$, the mean value of the amplitude of the baseband signal is $A \cdot d$. In practice, with a real recorded signal, the pulse amplitude is not constant over a long time. By normalizing the amplitude over a time $T$ corresponding to one pulse period with respect to the mean value and the duty cycle, the amplitude of the features passed to the HMM is independent of amplitude at the sensor output.

Figure 1(a) shows the block diagram for the normalization step. The signal $x_{f_{t}}(n)$ is broken into frames, $x_{b}(i, j)$, of length $T$ where $i$ is the frame index and $j$ is the sample index within the frame. The normalization is performed as

$$
\hat{x}_{b}(i, j)=\frac{x_{b}(i, j)}{\frac{1}{T \cdot d} \cdot \sum_{t=0}^{T-1} x_{b}(i, t)} .
$$

The duty cycle factor $d$ in the denominator before the summation in (3) is to normalize the amplitude of the pulses to unity. The last block reconstructs the framed signal into a continuous time signal.

Figure 2 shows an example of the feature extraction and normalization algorithm. The fullband signal is shown in figure 2(a). To demonstrate the effect of the normalization step, the fullband signal is increasingly scaled over time. The baseband signal is shown in figure 2(b), and the normalized baseband signal in figure 2(c). Both global and local pulse amplitude variations are normalized by the proposed method.

\section{HIDDEN MARKOV MODEL}

For the pattern detection and matching, a HMM is trained and used as described in [4] and [5]. The feature vectors used by the HMM are created by framing the signal $\hat{x}(n)$ as shown in figure $1(\mathrm{~b})$, where $\mathbf{o}(t)$ is the feature vector at time $t$.

\subsection{Training}

The training of the HMM can be stated as

$$
\lambda^{*}=\arg \max _{\lambda} P(\mathbf{O} \mid \lambda)
$$

where

$$
\mathbf{O}=\left\{\begin{array}{lll}
\mathbf{o}(0) & \mathbf{o}(1) & \cdots
\end{array}\right\}
$$

is a set of training feature vectors and

$$
\lambda=(\mathbf{A}, \mathbf{B}, \pi)
$$

are the parameters describing the hidden Markov model. The HMM parameters $\mathbf{A}, \mathbf{B}$ and $\pi$ are the state transition probability distribution, the observation symbol

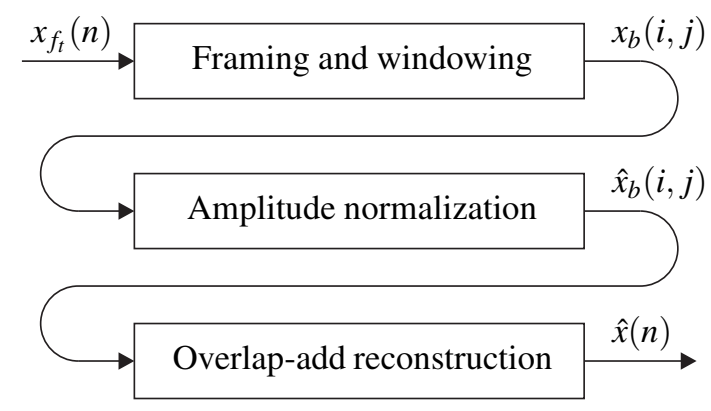

(a) Normalization of feature signal amplitude over time $T$. After framing and windowing, the signal pulses are scaled to unit amplitude and reconstructed.

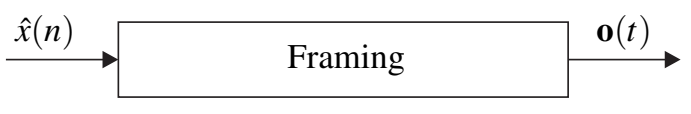

(b) Feature vector generation for the HMM.

Figure 1: Block diagrams for (a) feature normalization and (b) HMM feature vector generation.

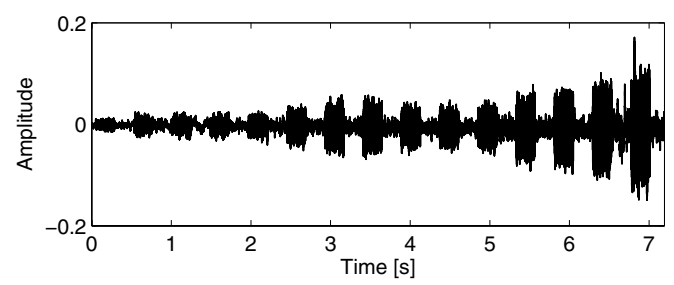

(a) Fullband signal $x(n)$. To demonstrate the effect of the normalization step, the fullband signal is increasingly scaled over time.

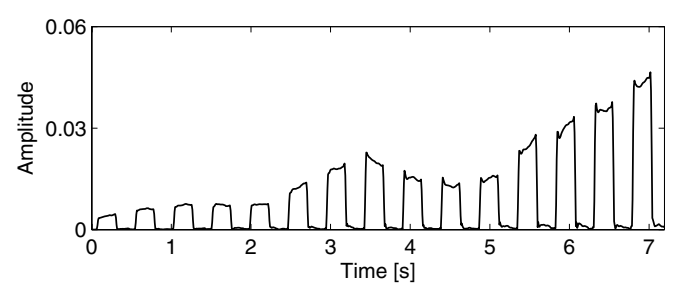

(b) Decimated baseband signal $x_{f_{t}}(n)$.

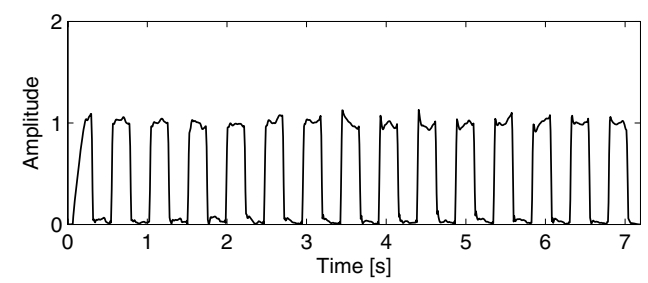

(c) Decimated baseband signal normalized, $\hat{x}_{f_{t}}(n)$, making the detection and matching amplitude independent.

Figure 2: Feature extraction. (a) shows the fullband signal, (b) the magnitude of the baseband signal and (c) the magnitude of the baseband signal when normalized over a pulse period. 


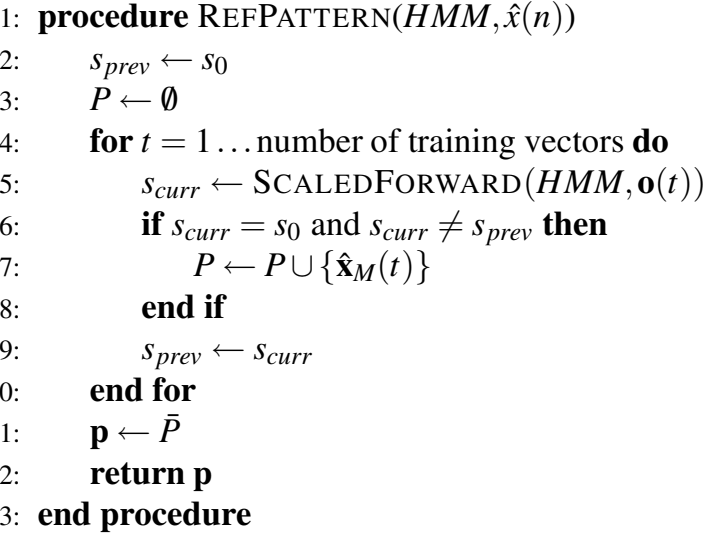

Figure 3: Proposed algorithm for calculating the reference pattern.

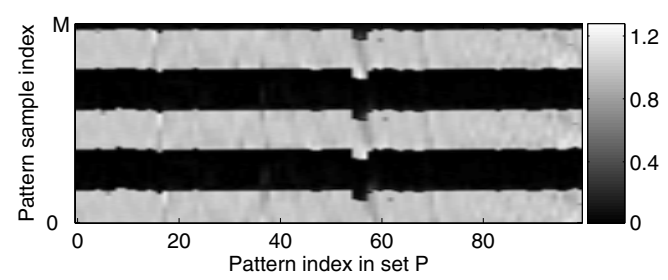

(a) The set $P$ of detected patterns in the training sequence.

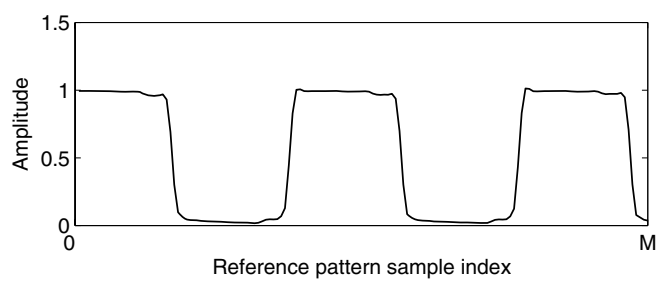

(b) Calculated reference pattern $\mathbf{p}$.

Figure 4: Calculation of reference pattern. Figure (a) is the set $P$ of all detected patterns in the training sequence, and (b) is the reference pattern $\mathbf{p}$ calculates as the average of all detected patterns.

probability distribution and the initial state distribution, respectively. Training the HMM is performed using the Baum-Welch reestimation method.

The feature vectors are created by framing the signal $\hat{x}(n)$ into blocks as

$$
\mathbf{o}(t)=\hat{\mathbf{x}}_{N}(t)
$$

where

$$
\hat{\mathbf{x}}_{N}(t)=\left[\begin{array}{lll}
\hat{x}(t-N+1) & \cdots & \hat{x}(t)
\end{array}\right]^{T}
$$

and where $N$ is chosen to 4 . The HMM is trained with two states as an ergodic model. The two states are expected to settle near the feature vectors with values of all 0's and all 1's. From here on the two expected states are denoted $s_{0}$ and $s_{1}$, respectively.

After training the HMM, a reference pattern for matching is calculated. The same training sequence is

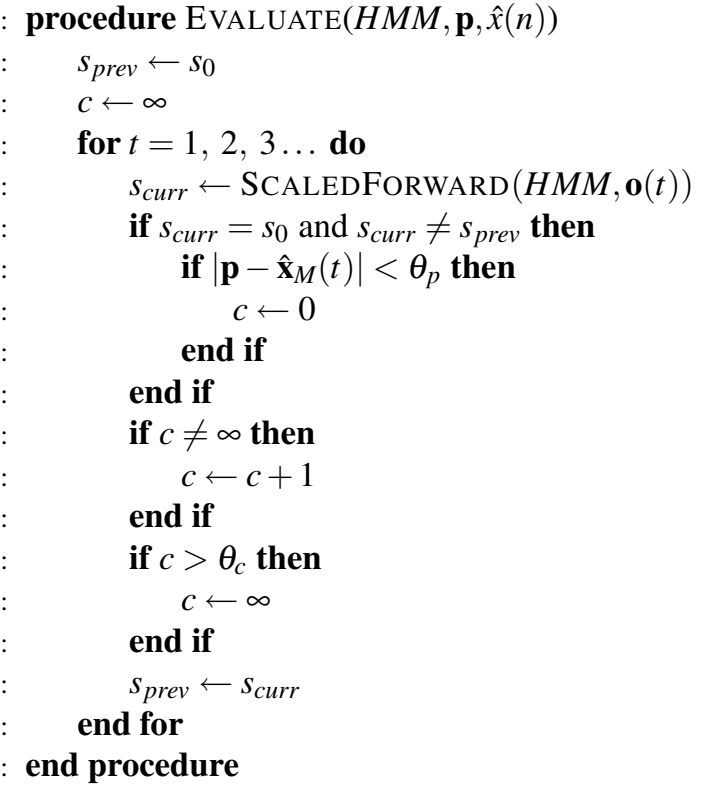

Figure 5: Proposed algorithm to determine the presence of an alarm signal.

passed through the HMM. On a transition from state $s_{1}$ to $s_{0}$ at a time $t, \hat{\mathbf{x}}_{M}(t)$ is saved for calculation of the reference pattern. The proposed algorithm in figure 3 shows how the reference pattern is calculated. The value of $M$ determines the length of the reference pattern, and is chosen to $M=2,5 \cdot T$.

The function SCALEDFORWARD, given a HMM and an observation vector, returns the most likely current state $s_{\text {curr }}$ (in [4], this is the index of the maximum value of the scaled state probability vector $\hat{\alpha}_{t}$, which is omitted for simplicity in the presented algorithm). The set $P$ contains all detected matching patterns, and the final reference pattern $\mathbf{p}$ is the average of all patterns in $P$.

Figure 4 shows the detected patterns and average pattern for the training sequence used during evaluation. The sequence is approximately 60 seconds long and the reverse alarm is on during the entire sequence. Figure 4(a) shows the set of all patterns detected during training. Figure 4(b) shows the average of all detected patterns, which is used as a reference pattern during the matching stage.

\subsection{Detection and Matching}

The procedure for detection and matching is similar to the one for the reference pattern calculation. The recorded sequence, after passing through the feature extraction and normalization, is passed through the HMM. On a state transition from $s_{1}$ to $s_{0}$ at time $t$, the reference pattern $\mathbf{p}$ is compared to $\hat{\mathbf{x}}_{M}(t)$. A threshold $\theta_{p}$ is used to determine whether the state transition was the result of noise or the presence of a pulse train pattern. Figure 5 shows the proposed algorithm for evaluating a signal and determine if and where a re- 
verse alarm is present.

The presence of a desired signal is determined using a counter, $c$. On a successful match against the reference pattern, the counter is reset to zero, and keeps increasing for each iteration. As long as the counter is below a specific threshold, $\theta_{c}$, the reverse alarm is considered active. As long as the reverse alarm is active, the counter will, after each state transition and successful match, keep being reset to zero. When the signal stops, it will reach its upper threshold and is set to infinity.

\section{EVALUATION}

To evaluate the proposed method, real recordings of a reverse alarm are used. The reverse alarm used in the evaluation is a J.Auer ESM Panel Mount Buzzer. The frequency is $3900 \mathrm{~Hz}$, pulse time $0,48 \mathrm{~s}$ and 0,5 duty cycle factor. The signals were sampled at $16 \mathrm{kHz}$, and a Hanning window with a $50 \%$ overlap is used in the framing and windowing step. The prototype lowpass filter is designed with $K=256$ and $L=4$.

Figure 6 and 7 shows the result from two recordings in two different environment. Figure 6 is recorded in a windy outdoor environment. The top graph shows the spectrogram of the signal, and the reverse alarm is clearly visible during three time periods at $3900 \mathrm{~Hz}$. The bottom graph shows the on and off state estimated by the proposed HMM and matching algorithm. Black color means no signal is detected $\left(c<\theta_{c}\right.$ in figure 5) and white color means a signal is detected. Figure 7 is recorded in an industrial environment. The noise levels are higher than in figure 6 and consists of noise from industrial machinery and vehicles. The reverse alarm is not as distinct as in figure 6 . In both figures there are three time periods where the reverse alarm is present, and in both cases the algorithm detects all three. Also, no false detections were made when the signal was not present.

A delay between the reverse alarm signal in the spectrogram and the estimated on and off state can be observed in the figures. This delay is due to the size of the reference pattern, where the whole reference pattern must be matched before the on state is assumed. With the reverse alarm used in the evaluation, this corresponds to approximately 1,25 seconds.

\section{CONCLUSION}

The proposed algorithm is implemented on a realtime platform. All steps involved in executing the algorithm for detection are numerically simple and suitable for realtime implementation on embedded platforms. Further tests will be performed to analyse the influence of stronger wideband noise, tonal noise near $f_{t}$ and impulse noise.

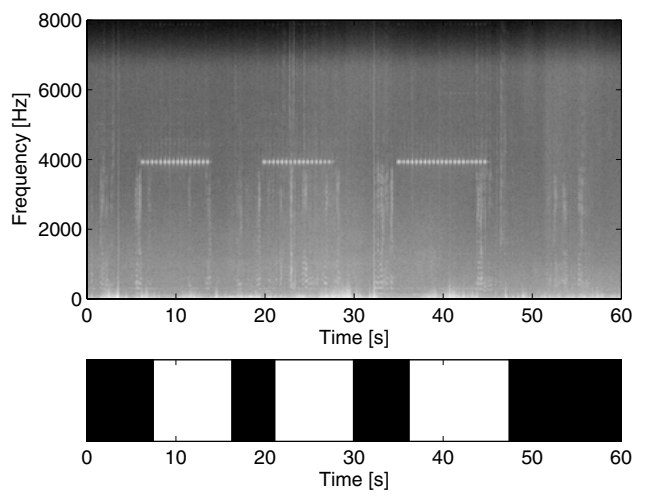

Figure 6: Outdoor recording.

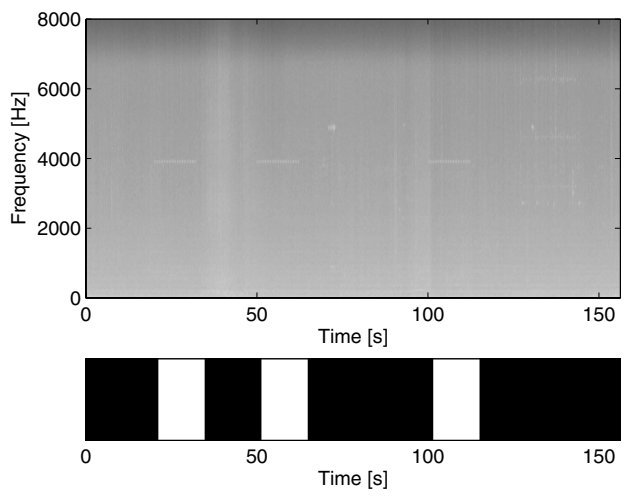

Figure 7: Recording in an industrial environment.

\section{REFERENCES}

[1] Brian D. Simpson, Robert S. Bolia, Richard L. McKinley, and Douglas S. Brungart. The impact of hearing protection on sound localization and orienting behavior. Human Factors, 47(1):188198, 2005.

[2] P. P. Vaidyanathan. Multirate systems and filter banks. Prentice Hall, 1993.

[3] Jan Mark de Haan, Nedelko Grbić, Ingvar Claesson, and Sven Nordholm. Filter bank design for subband adaptive microphone arrays. IEEE Transaction on Speech and Audio Processing, 11(1):14-23, January 2003.

[4] Lawrence Rabiner and Biing-Hwang Juang. Fundamentals of speech recognition. Prentice Hall, 1993.

[5] Mikael Nilsson. First order hidden markov model - theory and implementation issues. Technical report, Department of Signal Processing, Blekinge Institute of Technology, 2005. 